\title{
Fetal stem cell transplantation and gene therapy
}

Rachel Sagar (1), Cecilia Götherström (2), Anna L. David (1,3), Magnus Westgren (2)

1. Institute for Women's Health University College London London UK

2. Department of Clinical Science, Intervention and Technology, K57, Division of Obstetrics and Gynecology, Karolinska University Hospital Huddinge Karolinska Institutet Stockholm Sweden

3. Katholieke Universiteit LeuvenLeuvenBelgium

Correspondence;

M Westgren, MD, PhD, Senior Professor

K57, CLINTEC

Karolinska Institutet

14186 Stockholm

Sweden 


\section{Introduction}

Solid progress has been made in recent years with regards to fetal intervention for congenital conditions. Aside from widely practised fetoscopic laser coagulation in twin to twin transfusion syndrome, interventions for congenital diaphragmatic hernia and spina bifida, for example, are now being introduced into routine clinical care. However, by their very nature these surgical interventions are restricted to treating anatomical problems. The widespread availability of high resolution ultrasound scanning and the rapid advances in prenatal molecular diagnostic techniques, particularly testing for cell free fetal DNA in the maternal blood, means that congenital disorders are increasingly diagnosed early in gestation. In utero stem cell transplantation offer the promise of treatment, or even cure, for such genetic disorders. For in utero treatment to be contemplated, it must be possible to carry out the treatment safely for both mother and fetus, and must present advantages over postnatal treatment. Previous attempts to treat haematopoietic diseases with in utero transplantation of haematopoietic stem cells have failed for a variety of reasons. Combining genetic manipulation using gene therapy with fetal stem cell transplantation may be an alternative. Here we discuss the rationale for in utero stem cell transplantation alone or in combination with gene therapy, the ontological opportunities afforded by prenatal treatment and conversely the barriers to its success. We review the clinical evidence to date, highlighting recent breakthroughs in the journey from laboratory to clinical therapy. We lastly present two examples of clinical trials on in utero stem cell transplantation that are about to start recruitment.

\section{Rationale}

Much attention in the field of regenerative medicine has previously been focussed on haematopoietic stem cells (HSCs) with many thousands of patients treated for congenital disease and cancers using HSC transplantation in combination with myeloablation and immunosuppression therapy. More recently, the potential clinical utility of mesenchymal stem cells (MSCs) has also come to light. Collectively, these multipotent progenitor cells have been proposed as potential treatments for diseases ranging from severe combined immunodeficiencies (SCID) to osteogenesis imperfecta (OI). Regardless of stem cell classification or proposed target disease, the rationale for prenatal treatment is to improve the outcome for babies by beginning treatment before birth rather than waiting for postnatal intervention. Moreover, ameliorating pathology that has begun during fetal life, thus avoiding irreparable damage, may improve the transition from fetal to neonatal life and improve perinatal survival.

There are numerous physiological advantages of the unique in utero situation which support a prenatal approach. The average weight of a 20 week fetus is $300 \mathrm{~g}$, over 10 times smaller than the average term birthweight (Ghosh et al. 2018). There is therefore the opportunity for greatly increased cell dosage given the size and weight of the fetus compared with the neonate. This also brings a cost advantage, which is particularly important in the case stem cell or gene therapy biological products that are costly to manufacture. The presence of the foramen ovale and the ductus arteriosus within the fetal circulation permit therapeutic infusions into the umbilical vein to bypass the lungs, avoiding the sequestration in the lung microvasculature which occurs in postnatal infusion (Gholamrezanezhad et al. 2011; Erkers et al. 2015). Moreover, fetal life is a time of natural stem cell proliferation and migration to different anatomic compartments, so the developmental stage of the fetus provides an 
opportunity for wider expansion, migration and distribution of donor cells within the favourable fetal microenvironment (Taylor et al. 2016). Furthermore, the relatively naive fetal immune system may permit the development of donor specific immune tolerance. During fetal life, the immune system undergoes self-education, developing the ability to distinguish between autologous and foreign antigens. If transplantation occurs early enough, the foreign cells may be recognized as self and not undergo rejection. Not only does this permit acceptance of the graft without myeloablation or immunosuppression which is required for postnatal transplantation, it may also enable postnatal 'booster' transplantation with the same donor cells (Hayashi et al. 2002). Finally, instead of receiving advice that the only options are to terminate the pregnancy or await the delivery of a severely affected child, the prospect of an active prenatal approach may offer additional psychological benefits to parents whose fetus has a congenital disorder.

\section{Safety}

In utero stem cell transplantation is preferably achieved via a single injection, ideally into the umbilical vein at the intrahepatic portion or placental cord insertion which is a route that is commonly used in fetal medicine practice globally to transfuse the fetus with anaeamia. There are no large studies reporting on the risks of prenatal stem cell transplantation. Individual case reports from as early as 1967 used such disparate routes of administration, cell types and conditions treated as to make it difficult to draw conclusions. Data from fetal blood transfusion is more robust and can be used as a guide for the potential procedural risks. A recent study of over 900 transfusions reported a procedure related overall complication rate of $1.2 \%$, with a fetal loss rate of $0.6 \%$ (Zwiers et al. 2017). Less is known about the risks of in utero stem cell transplantation to the mother or her future progeny particularly in relation to potential transfer of cells into her circulation, and immune reactions. Further research is required in this area.

\section{Mesenchymal stem cells}

In 1966, a subset of cells within the bone marrow was found to have multilineage differentiation capacity. Due to their mesenchymal plasticity and potential use in bone repair, the term mesenchymal stem cell (MSC) was introduced in 1991. MSCs are a heterogenous group, with many potential fetal (for eg. fetal tissues and amniotic fluid), perinatal (for eg. placenta, umbilical cord and umbilical cord blood) and adult (for eg. bone marrow and adipose tissue) sources. MSC are multipotent stromal cells capable of differentiation into mesodermal lineages such as bone, cartilage, muscle and adipose (Pittinger et al 1999). The minimal criteria used for definition of in vitro cultured adult bone marrow-derived MSC are that they must be plastic-adherent when maintained in standard culture conditions, express CD105, CD73 and CD90, and be capable of in vitro differentiation into osteoblasts, adipocytes and chondroblasts (Dominici et al. 2006). There is no single surface marker to identify MSC. MSCs intrinsically migrate towards sites of injury in a CXCR4 dependent manner, delivering donor cells to target tissues, a characteristic which makes them particularly suitable for cellular therapy (Granero-Moltó et al. 2009). They are capable of engraftment and differentiation into specific cellular phenotypes. They also secrete bioactive factors which influence regeneration and turnover (Caplan \& Dennis 2006). Given their ability to secrete anti-apoptotic, anti-inflammatory, proangiogenic, pro-mitogenic and antibacterial factors, MSCs have been referred to as 'injury drugstores', capable of establishing a regenerative microenvironment to support the regeneration of injured tissue, whilst inhibiting recruitment 
of immune cells which could cause further damage (Caplan \& Correa 2011). Unlike haematopoietic stem cells (HSCS), MSCs have a low immunogenic profile and do not express HLA class II antigens, permitting cell infusion across major histocompatibility barriers without immunosuppression (Götherström 2004).

MSCs have been tested in clinical trials for a diverse variety of disorders ranging from Alzheimers disease to diabetes. Since the first clinical infusion was performed over twenty years ago, thousands of patients have received treatment, with few reports of infusion toxicity or serious adverse events adverse events documented (Lazarus et al. 1995; Squillaro et al. 2016). A 2012 meta-analysis of the randomised clinical trials did not detect an association with acute administration toxicity, organ system complications, infection, death, or malignancy although a significant association between MSC and transient fever was observed (Lalu et al. 2012). Most importantly, there have been no reports of ectopic tissue formation or malignant transformation in vivo, with laboratory findings confirming that MSC emerge from senescence at a frequency of much less than $10^{-9}$ ( Prockop et al. 2010). Whilst MSCs have been trialled for many conditions in adults, there are only two published case reports of prenatal MSC infusion, both for fetuses with OI. Two further cases of in utero MSC infusion for fetuses with OI are reported to have been carried out by a group in Italy, but no further information is published about these cases (Lanfranchi et al. 2009).

The first patient with type III OI reported in (Le Blanc et al. 2005; Götherström et al. 2014) received an ultrasound guided infusion of $6.5 \times 10^{6}$ fetal liver derived MSC into the intrahepatic umbilical vein at 32 weeks of gestation. There was no evidence of fetal distress during the procedure, and the remainder of the pregnancy was uneventful until spontaneous preterm rupture of the membranes at 35 weeks. She was delivered in good condition by caesarean section. At birth Wormian skull bones, generalised osteopenia, platyspondylia, thin gracile bowed long bones with deformities indicating healed fractures, and an actual fracture of the right femoral diaphysis were diagnosed, which is typical for severely affected OI neonates. Over the following 6 years, she clinically did better than would be expected with an average of 1 fracture and vertebral compression fracture per year, and growth along her own curve at 5 SD below the mean. Interestingly, she had low levels of donor cell engraftment (range between 0.3 and $16.6 \%$ ) in the bone at 9 months of age and no detectable engraftment aged 6 years. Between 6 and 8 years of age her growth dropped to 6.5 SD below the mean, instigating a second transplant of $2.8 \times 10^{6} / \mathrm{kg}$ same donor MSCs, with no adverse reactions recorded. Following this 'booster', her growth, fracture rate (no fractures the following 2 years) and ability to walk improved, despite a very low level of engraftment. Only 3 other individuals in the world are currently known to have an identical causative mutation in the COL1A2 gene. Little is known about these fetuses and children or their care, but they have been reported as suffering from type II/III OI, and one child died of the disease at 5 months of age (Götherström et al. 2014).

The second patient had a family history of type IV OI, and had ultrasound findings consistent with the diagnosis. She underwent allogeneic ultrasound guided intrahepatic umbilical vein infusion of $30 \times 10^{6}$ fetal liver MSC/kg at 31 weeks of gestation, with no acute complications. The remainder of the pregnancy was uneventful, with no new fractures detected. She was delivered in good condition at 38 weeks by elective caesarean section. Her postnatal course was promising, with no fractures experienced in the first year of life, whilst following her own 
growth curve just below the $3^{\text {rd }}$ percentile. Her longitudinal length plateaued aged 1 , hence a postnatal transplantation with $10 \times 10^{6}$ same donor $\mathrm{MSCs} / \mathrm{kg}$ was performed at nineteen months of age. No acute adverse events were observed, her growth subsequently continued just below the third percentile and she started to walk shortly after the transplantation. It has not been possible to investigate engraftment of donor cells. There are no published reports of patients with an identical mutation in the COL1A2 gene. However, four family members were found to have the same mutation, and have all experienced short stature and multiple fractures throughout childhood. Given the heterogeneity of type IV OI and the absence of other published cases with identical mutations it is difficult to conclude any efficacy of treatment, however, the return to normal growth following postnatal transplantation would again suggest some effect of MSC transplantation.

Importantly, in neither patient was there any evidence of immune reaction toward the donor cells, no development of donor specific antibodies, and no signs of acute or chronic complications, supporting the safety of MSC for prenatal transfusion.

Preclinical work has reinforced the potential of prenatal MSC transplantation as a treatment for OI. In neonatal oim mice, a naturally occurring model for severe type III OI, donor mouse adult MSCs have been shown to home to bone, engraft, differentiate and result in improved bone properties ( $\mathrm{Li}$ et al. 2007). In the Brtl/+ mouse model resembling moderately severe and lethal OI, prenatally transplanted mouse allogeneic adult whole bone marrow cells eliminated perinatal lethality and improved the mechanical properties of bones such that they were similar to wild type, supporting in utero transplantation as a promising approach for treatment (Panaroni et al. 2009). Transplanting human fetal MSC into oim mice resulted in considerable phenotypic improvement, with a two thirds reduction in long bone fracture rate and higher donor cell engraftment (highest in bone) than reported in other studies, coinciding with increased bone strength, thickness and length, suggesting that fetal to fetal transplantation may be more effective than using adult donor cells (Guillot et al. 2008; Vanleene et al. 2011).

Interestingly, small numbers of donor cells resulted in a large amount of healthy collagen in one OI mouse study: $2 \%$ engrafted donor cells led to $20 \%$ healthy collagen in the bones (Panaroni et al. 2009). Nevertheless, low engraftment levels in both murine experiments and in clinical cases reported have caused researchers to question the early concept of direct cell replacement being solely responsible for the improved clinical and bone mechanical properties seen following MSC transplantation. More recently, it has been proposed that the release of a soluble mediator from donor cells may explain the clinical effect seen despite low levels of engraftment (Otsuru et al. 2012; Ranzoni et al. 2016). MSC are known to secrete bioactive factors which influence cellular regeneration and turnover (Caplan \& Dennis 2006). It is this "MSC secretome" consisting of a wide range of factors such as cytokines, chemokines and growth factors, which is capable of having trophic effects on target tissues (Doorn et al. 2012). These factors are capable of modifying the microenvironment, stimulating endogenous cell proliferation and preventing apoptosis of resident cells (Caplan \& Dennis 2006). MSCs not only release cytokines, but also release large numbers of extracellular vesicles; membrane-derived bodies containing miRNA, mRNA, and proteins that are involved in cell-to-cell communication (Keshtkar et al. 2018). These paracrine effects could explain the therapeutic benefits seen even when MSC engraftment levels are low. 


\section{Clinical trial with MSCs}

The Boost Brittle Bones Before Birth (BOOSTB4) is an exploratory, open label multiple dose multicentre phase $\mathrm{I} / \mathrm{Il}$ trial evaluating safety and efficacy of postnatal or prenatal and postnatal infusions of allogeneic fetal liver-derived MSCs for the treatment of severe Ol compared with historical and untreated prospective controls.

The primary objective of the trial is to assess safety and tolerability in the woman, fetus and child after multiple intravenous infusions of allogeneic fetal MSC. It involves investigation of the seriousness, severity and frequency of treatment-related adverse events in the subjects. The follow-up is at 6 and 12 months after the last MSC infusion, but safety will be evaluated long-term to 10 years after the first dose.

No formal power calculations have been performed for the BOOSTB4 trial. OI is a rare disease and a limited number of subjects can be included each year. Based on the incidence, 30 patients will be included in this first study on the grounds of feasibility, suitable time period and for the application of descriptive statistical analyses.

Three populations will be studied under this protocol:

A. Infants diagnosed with OI type III or severe type IV on clinical grounds with a confirmatory sequence variant (mutation) in one or other of the type I collagen genes (COL1A1 and COL1A2)

B. Pregnant woman whose fetus has been diagnosed with OI type III or severe type IV prenatally on ultrasound parameters with a confirmatory sequence variant (mutation) in one or other of the type I collagen genes (COL1A1 and COL1A2)

C. Controls diagnosed with OI type III or severe type IV on clinical grounds with a confirmatory sequence variant (mutation) in one or other of the type I collagen genes (COL1A1 and COL1A2):

a) Matched historical controls ( $n=30-150)$ will be identified from national registries

b) Prospective untreated controls ( $n=$ up to 30 ).

Only patients diagnosed with qualitative (Glycine) substitution in the collagen triple-helix encoding region of either the COL1A1 or COL1A2 gene will be included in the trial.

Parents/authorized representatives or pregnant women of children/fetuses diagnosed with severe Ol will be informed about the trial and asked to give consent to the child's participation. Screening will be performed according to the trial-specific inclusion/exclusion criteria for the specific population group. Subjects eligible for the treatment trial but not willing/able to participate will be asked for inclusion as untreated prospective controls after being informed about the trial as described above. For the historical controls, as required by the local Ethics regulations, parents/authorized representatives of children diagnosed with $\mathrm{OI}$ type III or severe OI type IV will be informed about the trial and asked to give consent to the child's participation in the trial as matched, untreated control. Screening will be performed according to the trial-specific inclusion/exclusion criteria for the historical group. The historical controls will be matched for Ol type, sex, age at onset of bisphosphonate therapy and closest in time. Where possible, match will be made on Ol mutation, center/country of treatment and birth weight. 
Subjects in the postnatal group will be included in the trial and receive the first dose as soon as possible after birth up to 12 months of corrected age. They will receive 3 additional doses at $+4,+8$ and +12 months after the $1^{\text {st }}$ dose with an evaluation at the same time points. Subjects in the prenatal group will be included in the trial and receive the first treatment prenatally (between 16-35+6 weeks of gestation) and then be treated and evaluated at +4 (or as soon as possible after birth), $+8,+12$ months after the first dose with follow up at +6 and +12 months after the last dose. Subsequently all subjects (all children and the mothers where infusion was performed during pregnancy) will be followed for at least 10 years after the first dose in a non-interventional evaluation performed at the routine follow-up visits at the subjects Ol clinic.

The clinical trial will be carried out with an Advanced Therapy Medicinal Product (ATMP) product manufactured according to Good Manufacturing Practices (GMP)-standards at the accredited GMP-facility Vecura at Karolinska University Hospital in Sweden. The cryopreserved investigational medicinal product will be certified and released after fulfilment of a defined set of criteria. Ready-to-be-used investigational medicinal products will be shipped to the participating hospitals in Germany, the United Kingdom and the Netherlands outside Sweden. The investigational medicinal product will be administered as an intravenous infusion. Prenatal infusions will be made with a 20 Gauge $(0.7 \mathrm{~mm})$ spinal needle under ultrasound guidance into the intrahepatic portion of the umbilical vein.

A Data and Safety Monitoring Board is established and will follow the progress and quality of the trial. This board will review the general progress and feasibility of the trial, the quality and completeness of the data, and safety parameters. Adverse events will be monitored on an ongoing basis throughout the trial. The Data and Safety Monitoring Board will also perform pre-specified safety assessments within the trial. Also an external Scientific Advisory Board and an Ethical Advisory Board consisting of experts in the area overlook the study.

\section{Haematopoietic stem cells}

HSCs possess the key functional characteristics of self-renewal and of the capacity to generate all mature haematopoietic lineages, and are thus able to maintain functional haematopoiesis throughout life (Weissman \& Shizuru 2008). Congenital disorders of haematopoietic cells are common, and in particular affect low middle income countries eg haemoglobinopathies such as sickle cell or thalassaemia. The prospect of successful in utero transplantation resulting in lifelong correction of a wide range of disorders is therefore appealing. Whilst few cases of in utero MSC transplantation have been published, at least 46 prenatal HSC transplantations are documented in the literature. The first report of successful in utero haematopoietic transplantation was in a case of Bare Lymphocyte syndrome. A patient whose first child died of the condition underwent cordocentesis to confirm her second child was affected. At 30 weeks $16 \times 10^{6}$ fetal liver haematopoietic cells and thymic cells were infused into the placental cord insertion. There were no adverse effects and the remainder of the pregnancy was uneventful until normal delivery at term. The diagnosis was confirmed at delivery but it was noted that some cells expressing HLA class I were present. By one month of age $10 \%$ of lymphocytes had normal expression of class I HLA of donor type, thus confirming engraftment. However, the child had no B cell development, and did undergo seven postnatal stem cell grafts (Touraine et al. 1989). Following this seminal case, a number of in utero transplantations for fetuses with severe combined immunodeficiencies (SCID) were 
performed (Touraine et al. 2004; Flake et al. 1996; Wengler et al. 1996; Pirovano et al. 2004; Diukman \& Golbus 1992; Westgren et al. 2002). Of these nine cases who underwent transfusion of HSC from paternal or maternal bone marrow, maternal blood or fetal liver, eight fetuses were born alive and appeared well, without evidence of graft versus host disease. However, severe combined immunodeficiency is a unique disorder that facilitates donor $\mathrm{T}$ cell survival and proliferation due to the lack of competition of host immune cells, which is not the case for other haematopoietic disorders. Additionally, the engraftment achieved in these cases has only ever reached split chimersim, with reconstitution of the $T$ cell lineage only.

Acting upon the initial promise of in utero HSC transplantation, infusions were performed for diseases such as the Thalassaemias, Chronic Granulamotous Disease, Haemophilia A, Hurlers Syndrome, Metachromatic Leukodystrophy and Niemann Pick Disease. Cell sources including blood or bone marrow donated from family members and allogeneic fetal blood and liver were injected intravenously or intraperitoneally, at gestational ages ranging from 11 to 34 weeks. A summary of all published cases can be found in (Westgren 2006) and will not be reviewed again here. Whilst few complications of the infusion procedures occurred, transplantations outside of the context of severe combined immunodeficiencies were not successful in treating the underlying disease. In utero HSC transplantation appeared unable to establish robust levels of engraftment in immunocompetent recipient fetuses (Flake \& Zanjani 1999).

The competitive barriers to engraftment have been investigated in murine, ovine and canine models. Initially, the normal mouse model proved difficult to engraft, showing it to be an appropriate model replicating the barriers with engraftment seen in human fetuses (Carrier et al. 1995; Carrier et al. 2000). It was found that donor chimerism levels over $1 \%$ could be achieved with intravascular injection of high doses of cells, and that these high levels resulted in tolerance across major histocompatibility complex barriers (McClain \& Flake 2016). However, despite these findings, only $30 \%$ of recipients were chimeric, suggesting an immune barrier following transplantation. Tracking studies revealed that whilst mice transplanted with allogeneic and congeneic donor cells both achieved high initial levels of engraftment, $70 \%$ of allogeneic recipients lost the engraftment by 3-5 weeks following transplantation. Meanwhile, all the congenic animals remained chimeric (Peranteau et al. 2007). This was explained by further work showing that when transplanted pups were placed with a foster mother, rather than their mother who had undergone in utero transplantation, all recipients maintained their chimerism. The immune barrier in the allogeneic pups was therefore a result of maternal alloimmunisation and transfer of alloantibodies in breast milk, causing an adaptive immune response in the pup and loss of chimerism.

The study also found that if maternal donor cells were used for transplantation, engraftment and tolerance were maintained (Merianos \& Tiblad 2009). Whilst the mouse model is useful for pre-clinical work, there are a number of key differences between murine and human pregnancy, and the importance of maternal immunization in human in utero transplantation remains to be elucidated. Until this question is answered, some authors have recommended that any human in utero HSC transplantation takes place with maternal cells in order to avoid 
maternal alloimmunisation and fetal adaptive immune response taking place (McClain \& Flake 2016).

\section{Combining in utero stem cell transplantation with gene therapy}

Using gene corrected autologous fetal stem cells is one way to avoid the immune response associated with allogeneic HSCs. Stem cells are available from a number of sources within the fetus including the blood, liver, amniotic fluid (AF) and placenta. Fetal liver or blood sampling in early gestation carries a significant risk of miscarriage and so is unlikely to be clinically feasible. The AF and placenta are also sources of HSCs that can be easily collected at amniocentesis or chorionic villus sampling for prenatal diagnosis, procedures that have a low fetal mortality. Human AFS cells have the potential to differentiate into a variety of cell types and can be easily transduced without altering their characteristics. The potential for this combination of autologous in utero stem cell transplantation with gene therapy has been shown in sheep pre-clinical experiments. Here, AF-derived mesenchymal or HSCs were collected from mid-trimester fetal sheep using ultrasound-guided amniocentesis, transduced with an integrating lentiviral vector and transplanted back into the donor fetus using ultrasound guided intraperitoneal injection. There was widespread cell migration and engraftment in a variety of organs including those that are mainly haematopoietic, and in the blood (Steven Shaw et al. 2011; Shaw et al. 2015). These findings in a large animal model support the concept for clinical translation to treat congenital haematopoietic diseases in utero. The advantages to this combination approach is that the autologous stem cells are likely to engraft long term and at higher levels in the donor fetus when compared to allogeneic. In addition the genetic manipulation of the autologous stem cells occurs outside the fetus, avoiding the risk of germline gene transfer, off-targeting effects and transfer of the gene therapy to the mother (Ramachandra et al 2014). As only a relatively small number of stem cells are collected from the AF, this technique does rely on the ability to expand the HSC fraction while maintaining their haematopoietic differentiation potential. Nevertheless, in the sheep experiments, $10 \mathrm{ml}$ of AF yielded sufficient transduced AF-derived HSCs to reach engraftment levels of around 3\% in the fetal blood which might be sufficient to ameliorate severe disease (Shaw et al. 2015). More recent data comparing autologous with allogeneic mouse

The UK Gene Therapy Advisory Council (GTAC) considered that stem cell transplantation in utero offered therapeutic opportunities for a wide range of genetic disorders, and that such techniques were much more likely to be used in the short term than fetal gene therapy. They also considered an in utero stem cell gene therapy approach in their broader judgments about fetal gene therapy. The New and Emerging Technologies subgroup of GTAC found that the use of genetically modified stem cells in stem cell transplantation to the fetus was a possibility stating "such ex vivo modification would be unlikely to carry with it any higher risk to the germ line than the trials of postnatal somatic gene therapy which have already been approved" (Gene Therapy Advisory Council 1998).

\section{Clinical trial using HSC}

In utero HSC transplantation takes advantage of existing maternal-fetal tolerance during pregnancy, allowing the developing fetal immune system to induce tolerance to the transplanted cells without using conditioning or immunosuppression. To evaluate the safety, efficacy, and feasibility of this approach, the University of California, San 
Francisco (UCSF) in the United States is planning to perform a phase 1 clinical trial in fetuses with alpha thalassemia major and other similar variants. Alpha thalassemia major or Hemoglobin Bart's syndrome leads to severe fetal anaema which is fatal if untreated in utero. Based on the understanding that the developing fetal immune system should be tolerant to maternal antigens, the proposed stem cell source for the trial is maternal bone marrow-derived HSCs. Transplants will be performed at the same time as a clinically indicated intrauterine blood transfusion. This single center, non-randomized study aims to recruit 10 patients with the primary objective to evaluate the maternal and fetal safety of this combined approach. The secondary objectives are to evaluate the feasibility_of the combined procedures of fetal blood and HSC transfusions, including the ability to harvest an adequate cell yield from the maternal subjects. The efficacy of this approach, as measured by the ability to detect chimerism of maternal donor HSC in the fetal circulation, will also be evaluated.

Maternal subjects will be on study for at least 30 days after delivery. Fetal subjects will be on study for one year after delivery. Maternal and fetal subject participation will include the duration of gestation, including and following blood transfusions in utero and IUHCT, and the perinatal period until discharge from the hospital.

Consenting pregnant women whose fetus has been diagnosed with alpha thalassemia major or another alpha thalassemia variants demonstrating prenatal presentation consistent with Hemoglobin Barts syndrome will be screened for eligibility according to the trial-specific inclusion/exclusion criteria. Study subject will comprise of male or female fetuses 18-25 weeks gestational age, with alpha thalassemia major or another alpha thalassemia variants demonstrating prenatal presentation consistent with Hemoglobin Barts syndrome. Confirmation of diagnosis by either chorionic villus sampling, amniocentesis, or cordocentesis, or by identification of parents as genetic carriers and sonographic evidence of fetal anemia. Parents must elect to pursue in utero blood transfusions, and undergo subsequent in utero blood transfusions for the remainder of gestation. Fetal subjects who have received prior in utero blood transfusions may be included.

The cellular product for transplantation is maternal HSC (CD34+ cells, selected using Miltenyi CliniMACS, similar to the protocol used for postnatal transplantation). A cell dose of $10^{7}-10^{9}$ CD34+ cells $/ \mathrm{kg}$ (plus $1 \% \mathrm{~T}$ cells added back) was chosen based on large animal data. The fetus will then continue to have blood transfusions as clinically indicated, usually every 3 weeks. While the HSC transplantation takes place at UCSF, previous and subsequent in utero blood transfusions can be performed at the patient's local institutions, if there is expertise with fetal transfusions.

There is a Data and Safety Monitoring Board both internally at UCSF and an External Advisory Board of experts in Pediatric Anesthesiology, Maternal-Fetal Medicine, Pediatric Medicine, Pediatric Hematology, Pediatric and Fetal Surgery, Pediatric Bone Marrow Transplantation, Pediatric Neurology, Neonatology, Nursing and Medical Ethics. These boards were created specifically for this trial to determine how to attribute any fetal complication or loss (particularly since the fetuses enrolled in the study are likely to have hydrops secondary to their underlying disease, which increases the risk of adverse fetal events). Adverse events will be monitored on an ongoing basis throughout the study. 
All patients are counseled in a non-directive fashion regarding the possible choices for the pregnancy, including pregnancy termination, no fetal therapy, fetal therapy with in utero blood transfusions alone, or participating in the trial. Patients are informed of all the potential risks of the HSC transplantation, including the possibility of no benefit (no detectable chimerism) after the transplantation. One possible outcome is low levels of chimerism that are not enough to cure the disease, but that may allow improved tolerance to maternal HSC, which would allow a postnatal "booster" transplantation with maternal cells. If there is a high enough level of maternal cell chimerism, this treatment could lead to a definitive therapy before birth and avoid the complications of lifelong transfusions or the toxicity of a bone marrow transplant.

\section{Discussion and final comments}

There are several methodological and ethical considerations that need to be addressed when considering fetal stem cell or gene therapy. Some of these issues are summarized in Table 1. There must be a high certainty in regard to the fetal diagnosis. Although that prenatal diagnostics has developed rapidly during the last decade due to improved ultrasound scanning it is important to consider that misclassification is a rather common phenomenon, and therefore it is a prerequisite that the diagnoses is based on molecular genetic methods. These tests need to be carried out in laboratories of adequate standards and performance. Preferably the prenatal natural course of the disease should be known. If intrauterine life is associated with gradually impaired condition like in alpha thalassemia (increased anemia) or OI (fractures and impaired growth) there might be a case for early fetal intervention. Conversely if the affected fetus is protected during fetal life the situation is different. Currently, we are lacking information on the natural course before birth on many prenatally diagnosed disorders since many of these fetuses are terminated early in pregnancy. In some congenital disorders like storage disorders it is highly likely that the fetuses are severely affected from a very early stage and therefore might be not suitable candidates for fetal stem cell therapy. Here direct gene therapy might prove to be an option (Massaro et al 2018).

Informed consent is complicated to provide in an optimal way. One must be aware of that information given during pregnancy and at this stage might create exaggerated or false hope, which encourage the consenter to give consent before appreciating the issues fully. This is of crucial importance and preferable information is provided by independent personnel and not the researchers. The situation is complex since the primary benefactor, the fetus is unable to consent and lacks legal standing. Since the counselling is delicate it is reasonable according to our opinion that the mother get an adequate time period between the counselling and their decision to undergo this type of experimental treatment. How much the father should be involved in the decision making is an open question and might differ between different societies in regard to moral and legal standards. Since the decision involves assessments of risk-benefit for not only the fetus but also for the mother, it is according to our opinion a decision that should be taken by the mother. In regard to risk assessments it is crucial that more information on potential risks are made available. Currently we can only extract this information from a scattered literature and extrapolate from experience with other invasive fetal procedures. The risk-benefit issues arise in particular in connection to stem cell and gene therapies. Is there a risk that the treatment will help children to survive to a very difficult life or can cells or gene products cross the placenta to the mother and potentially affect the 
mother's condition? Thus, these far reaching consequences need to be considered but are difficult to comprehend for the patient but also for the responsible physician. A special issue is the risk for the fetus and mother to get sensitized for foreign allogenic cells. Potentially this might lead to long-term consequences in regard to receiving similar treatments after birth or receiving organ transplants. Although the risks are minimal they still exist and should be kept in mind when prenatal stem cell therapies are considered.

It is usually desirable to perform the fetal therapies as early as possible. Hypothetically, invasive fetal therapies could be done already in the first trimester into the yolk sac, or into different fetal compartments. However, considering that a certain time is required for the diagnoses and that the counselling process should not be disturbed it is unlikely that interventions with current technologies can be carried out in the first trimester. From the second trimester needling of the fetus is feasible and several studies report rather low rates of complications.

Finally, stem cells are sensitive for handling and expansion (Sipp et al 2018). They should be developed in approved processes that comply with GMP and should meet the standards of ATMP products, and should be well defined. The research should have necessary ethics approval and clinical trials should be conducting in accordance with all regulatory authorities. Any adverse events, side effects, and long-term safety risks must be assessed and mitigated, since transplanted cells may remain for many years in patients' bodies, which includes the pregnant woman. Therefore, careful monitoring and extended follow-up of the child and his/hers mother who receive prenatal stem cell treatments are of outmost importance. Taken these prerequisites into consideration it is natural that this type of experimental fetal therapies could not be carried out without adequate resources in regard to laboratory and clinical facilities as well as funding, and require collaboration between different disciplinaries, and institutions. 


\section{Table 1}

\begin{tabular}{|l|}
\hline Requirements for fetal stem cell or gene therapy \\
\hline Certainty about the fetal diagnosis \\
\hline The prenatal and postnatal natural course of the disease should be known \\
\hline Physiological advantage to commence therapy before birth \\
\hline Appropriate time for independent counselling \\
\hline An adequate risk-benefit ratio for the fetus \\
\hline An adequate risk-benefit ratio for the mother and any future children \\
\hline Not impair the chance for the child to have postnatal treatment \\
\hline $\begin{array}{l}\text { Well defined manufacture of the stem cell or gene therapy product with regard to quality, } \\
\text { homogeneous cell population and expansion etc. }\end{array}$ \\
\hline $\begin{array}{l}\text { Feasible to deliver the stem cells or gene corrected cells during fetal life to target the } \\
\text { disease }\end{array}$ \\
\hline Systematic long term follow-up \\
\hline
\end{tabular}




\section{References}

Le Blanc, K. et al., 2005. Fetal mesenchymal stem-cell engraftment in bone after in utero transplantation in a patient with severe osteogenesis imperfecta. Transplantation, 79(11), pp.1607-1614.

Caplan, A.I. \& Correa, D., 2011. The MSC: an injury drugstore. Cell stem cell, 9(1), pp.11-5.

Caplan, A.I. \& Dennis, J.E., 2006. Mesenchymal stem cells as trophic mediators. Journal of Cellular Biochemistry, 98(5), pp.1076-1084.

Carrier, E. et al., 1995. Induction of Tolerance in Nondefective Mice After in-Utero Transplantation of Major Histocompatibility Complex-Mismatched Fetal Hematopoietic Stem-Cells. Blood, 86(12), pp.4681-4690.

Carrier, E. et al., 2000. Microchimerism does not induce tolerance after in utero transplantation and may lead to the development of alloreactivity. Journal of Laboratory and Clinical Medicine, 136(3), pp.224-235.

Diukman, R. \& Golbus, M.S., 1992. In utero stem cell therapy. The Journal of reproductive medicine, 37(6), pp.515-20.

Dominici, M. et al., 2006. Minimal criteria for defining multipotent mesenchymal stromal cells. The International Society for Cellular Therapy position statement. Cytotherapy.

Doorn, J. et al., 2012. Therapeutic Applications of Mesenchymal Stromal Cells: Paracrine Effects and Potential Improvements. Tissue Engineering Part B: Reviews, 18(2), pp.101115.

Erkers, T. et al., 2015. Treatment of severe chronic graft-versus-host disease with decidual stromal cells and tracing with (111)indium radiolabeling. Stem cells and development, 24(2), pp.253-63.

Flake, A.W. et al., 1996. Treatment of X-Linked Severe Combined Immunodeficiency by in Utero Transplantation of Paternal Bone Marrow. New England Journal of Medicine, 335(24), pp.1806-1810.

Flake, A.W. \& Zanjani, E.D., 1999. In Utero Hematopoietic Stem Cell Transplantation: Ontogenic Opportunities and Biologic Barriers. Blood, 94(7), p.2179 LP-2191.

Gene Therapy Advisory Council, 1998. Report on the Potential Use of Gene Therapy In Utero,

Gholamrezanezhad, A. et al., 2011. In vivo tracking of 111In-oxine labeled mesenchymal stem cells following infusion in patients with advanced cirrhosis. Nuclear Medicine and Biology, 38(7), pp.961-967.

Ghosh, R.E. et al., 2018. Birth weight trends in England and Wales (1986-2012): babies are getting heavier. Archives of disease in childhood. Fetal and neonatal edition, 103(3), pp.F264-F270.

Götherström, C., 2016. Human Foetal Mesenchymal Stem Cells. Best Practice and Research: Clinical Obstetrics and Gynaecology, 31, pp.82-87.

Götherström, C., 2004. Immunologic properties of human fetal mesenchymal stem cells. American Journal of Obstetrics and Gynecology, 190(1), pp.239-245.

Götherström, C. et al., 2014. Pre- and postnatal transplantation of fetal mesenchymal stem cells in osteogenesis imperfecta: a two-center experience. Stem cells translational medicine, 3(2), pp.255-64.

Granero-Moltó, F. et al., 2009. Regenerative effects of transplanted mesenchymal stem cells in fracture healing. Stem cells (Dayton, Ohio), 27(8), pp.1887-98.

Guillot, P. V. et al., 2008. Intrauterine transplantation of human fetal mesenchymal stem cells from first-trimester blood repairs bone and reduces fractures in osteogenesis imperfecta mice. Blood, 111(3), pp.1717-1725. 
Hayashi, S. et al., 2002. Complete allogeneic hematopoietic chimerism achieved by a combined strategy of in utero hematopoietic stem cell transplantation and postnatal donor lymphocyte infusion. Blood, 100(3), p.804 LP-812.

Keshtkar, S., Azarpira, N. \& Ghahremani, M.H., 2018. Mesenchymal stem cell-derived extracellular vesicles: novel frontiers in regenerative medicine. Stem cell research \& therapy, 9(1), p.63.

Lalu MM et al., 2012 Safety of cell therapy with mesenchymal stromal cells (SafeCell): a systematic review and meta-analysis of clinical trials.PLoS One. 7(10):e47559.

Lanfranchi, A., Porta, F. \& Chirico, G., 2009. Stem cells and the frontiers of neonatology.

Early Human Development, 85(10), pp.S15-S18.

Lazarus, H.M. et al., 1995. Ex vivo expansion and subsequent infusion of human bone marrow-derived stromal progenitor cells (mesenchymal progenitor cells): implications for therapeutic use. Bone marrow transplantation, 16(4), pp.557-64.

Li, F., Wang, X. \& Niyibizi, C., 2007. Distribution of Single-Cell Expanded Marrow Derived Progenitors in a Developing Mouse Model of Osteogenesis Imperfecta Following Systemic Transplantation. Stem Cells, 25(12), pp.3183-3193.

Massaro, G, et al.,(2018). Fetal gene therapy for neurodegenerative disease of infants. Nat Med. 2018 Sep;24(9):1317-1323

McClain, L.E. \& Flake, A.W., 2016. In utero stem cell transplantation and gene therapy: Recent progress and the potential for clinical application. Best Practice and Research: Clinical Obstetrics and Gynaecology, 31, pp.88-98.

Merianos, D. \& Tiblad, E., 2009. Maternal alloantibodies induce a postnatal immune response that limits engraftment following in utero hematopoietic cell transplantation in mice. The Journal of clinical inves 119(9), pp 2590-600.

Otsuru, S. et al., 2012. Transplanted bone marrow mononuclear cells and MSCs impart clinical benefit to children with osteogenesis imperfecta through different mechanisms. Blood, 120(9), pp.1933-1941.

Panaroni, C. et al., 2009. In utero transplantation of adult bone marrow decreases perinatal lethality and rescues the bone phenotype in the knockin murine model for classical, dominant osteogenesis imperfecta. Blood, 114(2), pp.459-468.

Peranteau, W.H. et al., 2007. Evidence for an immune barrier after in utero hematopoieticcell transplantation. Blood, 109(3), pp.1331-1333.

Pirovano, S. et al., 2004. Reconstitution of T-cell compartment after in utero stem cell transplantation: analysis of T-cell repertoire and thymic output. Haematologica, 89(4), pp.450-61.

Pittenger, MFet al. Multilineage potential of adult human mesenchymal stem cells. Science. 1999 Apr 2;284(5411):143-7

Prockop, D.J. et al., 2010. Defining the risks of mesenchymal stromal cell therapy. Cytotherapy, 12(5), pp.576-578.

Ramachandra DL et al., 2014 In utero therapy for congenital disorders using amniotic fluid stem cells. Front Pharmacol. Dec 19;5:270. doi: 10.3389/fphar.

Ranzoni, A.M. et al., 2016. Counteracting bone fragility with human amniotic mesenchymal stem cells. Scientific Reports, 6, p.39656.

Shaw, S.S.W. et al., 2015. Sheep CD34+ Amniotic Fluid Cells Have Hematopoietic Potential and Engraft After Autologous In Utero Transplantation. Stem Cells, 33, pp.122-132.

Squillaro, T., Peluso, G. \& Galderisi, U., 2016. Review Clinical Trials With Mesenchymal Stem Cells: An Update. Cell Transplantation, 25, pp.829-848. 
Sipp D et al., 2018 Clear up this stem-cell mess Nature $561,455-457$

Steven Shaw, S.W. et al., 2011. Autologous transplantation of amniotic fluid-derived mesenchymal stem cells into sheep fetuses. Cell Transplantation, 20, pp.1015-1031.

Taylor, P.A. et al., 2016. Brief report Allogenic fetal liver cells have a distinct competitive engraftment advantage over adult bone marrow cells when infused into fetal as compared with adult severe combined immunodeficient recipients. Blood, 99(5), pp.1870-1873.

Touraine, J.-L. et al., 2004. Reappraisal of in utero stem cell transplantation based on longterm results. Fetal diagnosis and therapy, 19(4), pp.305-12.

Touraine, J.L. et al., 1989. IN-UTERO TRANSPLANTATION OF STEM CELLS IN BARE LYMPHOCYTE SYNDROME. The Lancet, 333(8651), p.1382.

Vanleene, M. et al., 2011. Transplantation of human fetal blood stem cells in the osteogenesis imperfecta mouse leads to improvement in multiscale tissue properties. Blood, 117(3), pp.1053-1060.

Weissman, I.L. \& Shizuru, J.A., 2008. The origins of the identification and isolation of hematopoietic stem cells, and their capability to induce donor-specific transplantation tolerance and treat autoimmune diseases. Blood, 112(9), pp.3543-3553.

Wengler, G.S. et al., 1996. In-utero transplantation of parental CD34 haematopoietic progenitor cells in a patient with X-linked severe combined immunodeficiency (SCIDXI). Lancet (London, England), 348(9040), pp.1484-7.

Westgren, M., 2006. In utero stem cell transplantation. Semin Reprod Med, 24(5), pp.348357.

Westgren, M. et al., 2002. Prenatal T-cell reconstitution after in utero transplantation with fetal liver cells in a patient with X-linked severe combined immunodeficiency. American Journal of Obstetrics and Gynecology, 187(2), pp.475-482.

Zwiers, C. et al., 2017. Complications of intrauterine intravascular blood transfusion: lessons learned after 1678 procedures. Ultrasound in Obstetrics \& Gynecology, 50(2), pp.180186. 